\title{
Metaanalyse für ältere PCNSL-Patienten
}

\section{Die Inzidenz primärer Lymphome des Zentralnervensystems (PCNSL) nimmt zu. Jeder zweite Patient ist über 60 Jahre alt. Aber welche Behandlung ist die beste für die älteren Patienten mit PCNSL?}

$\mathrm{n}$ den vergangenen 30 Jahren sind PCNSL unter immunkompetenten $\mathrm{Pa}$ tienten immer häufiger geworden. Dabei handelt es sich um aggressive NonHodgkin-Lymphome (NHL), die ausschließlich das Zentralnervensystem befallen. Behandelt wird gemeinhin mit einer hochdosierten Chemotherapie auf Basis von Methotrexat (HD-Mtx) oder Cytarabin (HD-Cyt); hinzu kommt in jüngerer Zeit eine Ganzhirnbestrahlung zur Konsolidierung. Diese scheint nicht das Leben zu verlängern, aber eine bessere Tumorkontrolle zu ermöglichen.

Offen ist allerdings, wie man ältere $\mathrm{Pa}$ tienten behandeln sollte, obwohl rund $50 \%$ aller PCNSL-Patienten über 60 Jahre alt sind. Ein internationales Team hat deshalb in einem systematischen Review den Wert verschiedener Erstlinienthera- pien bei älteren immunkompetenten $\mathrm{Pa}$ tienten mit PCNSL untersucht. Darüber hinaus nahmen die Forscher eine Metaanalyse zusätzlicher individueller Patientendaten aus Studien und einer internationalen Kollaboration vor.

In Datenbanken wurden 20 auswertbare Studien zum Thema gefunden, 13 mit individuellen Daten für insgesamt 405 Patienten, die mit den Daten von 378 weiteren Patienten gepoolt werden konnten. Das mediane Alter der 783 Patienten lag bei 68 Jahren, der KarnofskyPerformance-Score (KPS) bei $60 \%$.

Die Behandlung der Patienten variierte stark: 573 (73\%) unterzogen sich eine rHD-Mtx-Therapie, 276 einer Ganzhirnbestrahlung mit median 36 Gy. Ein KPS $\geq 70 \%$ war der stärkste prognostische Faktor für die Mortalität mit einer Ha- zard Ratio [HR] von 0,50 . Nach median 40 Monaten Follow-up war eine HDMtx-Therapie mit einem besseren Überleben verbunden (HR 0,70). Es gab keinen Unterschied zwischen HD-Mtx plus oraler Chemotherapie und aggressiveren HD-Mtx-basierten Therapien (HR 1,39). Eine Radiotherapie war mit einem verbesserten Überleben, aber auch mit einem erheblich höheren Risiko für Neurotoxizität verbunden (Odds Ratio 5,23).

Fazit: Ältere Patienten mit PCNSL profitieren von einer HD-Mtx-Therapie vor allem, wenn sie mit oralen Alkylanzien kombiniert wird. Aggressivere Protokolle scheinen das Ergebnis nicht $\mathrm{zu}$ verbessern. Eine Ganzhirnbestrahlung könnte es verbessern, ist aber mit mehr Neurotoxizität verbunden. Prospektive Untersuchungen älterer PCNSL-Patienten sind nötig.

Christina Berndt

Kasenda B et al. First-line treatment and outcome of elderly patients with primary central nervous system lymphoma (PCNSL) - a systematic review and individual patient data meta-analysis. Ann Oncol. 2015;26(7):1305-13.

\section{Glioblastom: Bevacizumab in der Erstlinie - Vorteile bei proneuralen Tumoren}

\section{Beim Glioblastom hatte Bevacizumab in der First-Line zusätzlich zur Standard- therapie insgesamt keinen Effekt auf das Gesamtüberleben. Möglicherweise gibt es molekularbiologisch definierte Subgruppen, die doch profitieren.}

\footnotetext{
lioblastompatienten, die mit Strahlentherapie plus Temozolomid, behandelt werden, entwickeln oft schon sechs Monate später ein Rezidiv. In dieser Situation ist der anti-VEGF-Antikörper Bevacizumab als Mono- oder Kombinationstherapie zugelassen. In der Erstlinie hatte Bevacizumab in den beiden Phase-III-Studien AVAglio (Avastin in Glioblastoma) und RTOG-0825 zwar das progressionsfreie Intervall (PFS) im Vergleich zur Standardbehandlung verlängert, dieser Vorteil mündete jedoch nicht in einem signifikant besseren $\mathrm{Ge}$ samtüberleben. Die Suche nach Subgruppen, die besonders profitierten - gebildet anhand von klassischen Prognosefaktoren - verlief negativ. Nun suchten
}

Thomas Sandmann und Kollegen in der AVAglio-Studie nun nach molekular definierten Subtypen, die besonders sensitiv auf die Dreierkombination (Bevacizumab/Temozolomid/Radiotherapie reagierten. Von 349 Patienten der AVAglio-Studie standen prätherapeutisch gewonnene Tumorproben für die Biomarkeranalyse zur Verfügung (Bevacizumab-Arm: $\mathrm{n}=171$, Placeboarm: $\mathrm{n}=$ 178). Untersucht wurden der Mutationsstatus der Isocitratdehydrogenase 1 (IDH1) sowie das Genexpressionsprofil, das eine Zuordnung $u$. a. in die beiden Subgruppen mesenchymales und proneurales Glioblastom ermöglicht. Die Ergebnisse wurden mit den Überlebensdaten korreliert.
Im Vergleich zu Placebo ergab sich für Bevacizumab ein signifikanter Vorteil beim Gesamtüberleben für Patienten mit proneuralen IDH1-Wildtyp-Tumoren (17,1 vs. 12.8 Monate; Hazard Ratio $0,43 ; p=0,002)$. Zwar war auch bei Patienten mit mesenchymalen Tumoren ein Vorteil beim PFS unter Bevacizumab zu beobachten, auf das Gesamtüberleben hatte der anti-VEGF-Antikörper bei diesem Subtyp aber keinen Einfluss.

Fazit: Patienten mit proneuralem IDH1Wildtyp-Glioblastom profitieren wahrscheinlich im Gegensatz zu anderen Glioblastompatienten von Bevacizumab zusätzlich zur Erstlinien-Standardtherapie auch im Hinblick auf das Gesamtüberleben. Das sollte nun in einem unabhängigen Kollektiv validiert werden.

Barbara Kreutzkamp

Sandmann TS et al. Patients With Proneural Glioblastoma May Derive Overall Survival Benefit From the Addition of Bevacizumab to FirstLine Radiotherapy and Temozolomide: Retrospective Analysis of the AVAglio Trial J Clin Oncol. 2015;33(25):2735-44. 